\title{
High-Phosphorus Diet Induces Osteopontin Expression of Renal Tubules in Rats
}

\author{
Hiroshi Matsuzaki ${ }^{1, *}$, Shin-ichi Katsumata ${ }^{2}$, Mariko Uehara ${ }^{2}$, Kazuharu Suzuki², \\ and Misao Miwa ${ }^{1}$ \\ ${ }^{1}$ Department of Nutrition, Junior College of Tokyo University of Agriculture, 1-1-1 Sakuragaoka, Setagaya-ku, \\ Tokyo 156-8502, Japan \\ ${ }^{2}$ Department of Nutritional Science, Faculty of Applied Bioscience, Tokyo University of Agriculture, 1-1-1 \\ Sakuragaoka, Setagaya-ku, Tokyo 156-8502, Japan
}

Received 29 January, 2007; Accepted 2 April, 2007

\begin{abstract}
Summary High-phosphorus $(\mathrm{P})$ diet induces nephrocalcinosis in rats; however, the mechanism for onset of this disorder is unclear. The calcium (Ca) deposits in kidney are a form of hydroxyapatite, while osteopontin is combined with hydroxyapatite. Based on these observations, we speculated that the osteopontin play an important role in the formation of the Ca deposits induced by high-P diet. This study was investigated the effect of high-P diet on osteopontin expression in kidney. Female Wistar rats were fed diets containing $P$ concentrations of either $0.3 \%$ (control diet) or $1.5 \%$ (high-P diet) for 14 days. On von Kossa staining, Ca deposits were seen in the tubules of the cortex, outer medulla and inner medulla in rats fed on the high-P diet. Expression of osteopontin was confirmed in rats fed on the high-P diet by immunohistochemical staining, and the localization of this protein was in the same region as the Ca deposits. On the other hand, no evidence of $\mathrm{Ca}$ deposits and osteopontin expression was observed in the tubules of the cortex, outer medulla or inner medulla of rats fed on the control diet. These results suggest that high-P diet induces osteopontin expression in the renal tubules. Moreover, our results suggest that increase in osteopontin expression in the renal tubules is presumably involved in the formation of $\mathrm{Ca}$ deposits induced by high-P diet.
\end{abstract}

Key Words: osteopontin, calcium deposits, high-phosphorus diet, rats

\section{Introduction}

Nephrocalcinosis is a disorder involving calcium $(\mathrm{Ca})$ deposits in the rat kidney. It is known that dietary phosphorus (P) concentration is an important etiologic factor in nephrocalcinosis. It has also been reported that rats fed high-P diet display increased kidney $\mathrm{Ca}$ and $\mathrm{P}$ concentrations and development of nephrocalcinosis on histological examination

\footnotetext{
*To whom correspondence should be addressed.

Tel: +81-3-5477-2582

E-mail: matsu@nodai.ac.jp
}

[1-5]. Thus, although large numbers of studies have been conducted, the effects of high-P diet on nephrocalcinosis and the mechanisms responsible for onset of this disorder remain unclear. To prevent this disorder, better understanding of these issues is needed, particularly with regard to nutritional research using rodents, as the onset of nephrocalcinosis may complicate the interpretation of experimental results.

With regard to the mechanism responsible for onset of nephrocalcinosis, we hypothesize that during the formation of $\mathrm{Ca}$ deposits, matrix ingredients initially act as a nucleus, around which $\mathrm{Ca}$ deposits are formed. In other words, matrix ingredients are important factors in the formation of $\mathrm{Ca}$ deposits induced by a high-P diet. However, no studies have 
examined the relationship between matrix ingredients and $\mathrm{Ca}$ deposits in rats fed a high-P diet. X-ray microanalysis has revealed that the major constituents of $\mathrm{Ca}$ deposits are $\mathrm{Ca}$ and $\mathrm{P}$, probably in the form of hydroxyapatite $[6,7]$. Our previous electron microscopy studies $[7,8]$ also confirmed that deposition of hydroxyapatite occurred in the renal tubules of rats fed high-P diet. While, osteopontin binds extremely tightly to hydroxyapatite [9]. Based on these observations, we speculated that the osteopontin as matrix components play an important role in the formation of the Ca deposits induced by high-P diet. Accordingly, this study investigated whether high-P diet affects osteopontin expression in renal tubules as evaluated using immunohistochemical method.

\section{Materials and Methods}

\section{Animals and diets}

Four-week-old female Wistar rats (Clea Japan, Tokyo, Japan) were housed in individual stainless-steel wire-mesh cages. During the experiment, cages were located in a room with controlled lighting under a 12-h light:dark cycle (light, $0800-2000 \mathrm{~h}$ ) at a temperature of $22 \pm 1^{\circ} \mathrm{C}$ and relative humidity of $60-65 \%$. The compositions of the experimental diets are shown in Table 1. Experimental diets were based on AIN-93G diet [10]. Two experimental diets containing the two different $\mathrm{P}$ concentrations (control diet, 0.3\%; high$\mathrm{P}$ diet, 1.5\%). Before the study period began, there was a 7 day acclimatization period during which all rats were given free access to control diet and deionized water. After the

Table 1. Composition of the experimental diets.

\begin{tabular}{lcc}
\hline & Control diet & High-P diet \\
\hline Ingredient & \multicolumn{2}{c}{$\mathrm{g} / \mathrm{kg}$} \\
Corn starch & 529.486 & 476.763 \\
Casein & 200.0 & 200.0 \\
Sucrose & 100.0 & 100.0 \\
Soybean oil & 70.0 & 70.0 \\
Cellulose powder & 50.0 & 50.0 \\
Mineral mix ${ }^{1}$ & 35.0 & 35.0 \\
Vitamin mix ${ }^{2}$ & 10.0 & 10.0 \\
L-Cystine & 3.0 & 3.0 \\
Choline bitartrate & 2.5 & 2.5 \\
Tert-butylhydroquinone & 0.014 & 0.014 \\
KH ${ }_{2}$ PO $_{4}$ & - & 52.723 \\
\hline Chemical analysis & & \\
Ca & 0.50 & 0.51 \\
Mg & 0.048 & 0.046 \\
$\mathrm{P}$ & 0.31 & 1.54
\end{tabular}

${ }^{1}$ AIN-93G mineral mix.

${ }^{2}$ AIN-93 vitamin mix. acclimatization period, rats were randomly divided into two groups and were fed the diets with different $\mathrm{P}$ concentrations for 14 days. Rats fed the control diet were given the weight of food consumed by the rats fed the high-P diet on the previous day. Rats were given free access to deionized water. At the end of the experimental period, rats were sacrificed, and both kidneys were collected for analysis. The left kidney was used for mineral analysis, and the right was used for histological studies. Animals were treated in accordance with the guidelines of the National Research Council for the Care and Use of Laboratory Animals (1985).

\section{Mineral analysis of experimental diet and kidney}

The left kidney was weighed after the renal capsule was discarded. After drying overnight at $100^{\circ} \mathrm{C}$, and the dry weight was measured. Samples of the experimental diets and kidney were ashed at $550^{\circ} \mathrm{C}$ for $48 \mathrm{~h}$ in a muffle furnace, and minerals were extracted in $1 \mathrm{~mol} / \mathrm{L}$ of $\mathrm{HCl}$ for analysis. $\mathrm{Ca}$ and magnesium $(\mathrm{Mg})$ were determined by atomic absorption spectrometry (Hitachi A-2000) [11]. P was analyzed colorimetrically according to the method of Gomori [12].

\section{Histological examination}

Von Kossa staining. Immediately after the right kidney was removed, the kidney was fixed in 4\% paraformaldehyde (PFA) for subsequent processing and paraffin embedding following routine methods for histopathology. Sections $(3 \mu \mathrm{m})$ were cut and stained with von Kossa's solution.

Immunohistochemical staining. As described above, PFA-fixed, paraffin-embedded sections $(3 \mu \mathrm{m})$. Sections were deparaffinized in xylene, rehydrated in a graded ethanol series, and endogenous peroxidase activity was blocked with $0.3 \%$ hydrogen peroxide in distilled water. For antigen retrieval, sections were incubated at high temperature $\left(120^{\circ} \mathrm{C}\right.$ for $\left.10 \mathrm{~min}\right)$ for antigen unmasking in $0.01 \mathrm{~mol} / \mathrm{L}$ citrate buffer ( $\mathrm{pH}$ 6.0). Non-specific binding sites were blocked with skim milk solution. Sections were stained for $4^{\circ} \mathrm{C}$ for $1 \mathrm{~h}$ with Anti (mouse MAP) Osteopontin (1:100; LSL Co., Japan). After washing in phosphatebuffered saline ( $\mathrm{pH}$ 7.4), Labeled-treptavidin-biotin-peroxidase complex (LSAB system, DAKO Cytomation., Japan) was applied, and sections were visualized with diaminobenzidine. Nuclear counter-staining was performed with Mayer's hematoxyline.

\section{Statistical analysis}

Results are presented as means $\pm \mathrm{SD}$. After conducting an F-test to determine the homogeneity of variances, Student's $t$ test was used to determine significant differences between the two groups. If the homogeneity of variances was not equal, Welch's $t$ test was used instead of Student's $t$ test. Differences were considered significant at $p<0.05$. 
Table 2. Body weight in rats fed on control and high-P diet.

\begin{tabular}{lcc}
\hline & Control diet & High-P diet \\
\hline Initial body weight $(\mathrm{g})$ & $84.6 \pm 3.9$ & $84.4 \pm 3.6$ \\
Final body weight $(\mathrm{g})$ & $132.1 \pm 4.1$ & $132.9 \pm 6.5$ \\
\hline
\end{tabular}

Values are means $\pm \mathrm{SD}, n=5$ per group.

Table 3. Kidney mineral concentration in rats fed on control and high-P diet.

\begin{tabular}{lrr}
\hline & Control diet & \multicolumn{1}{c}{ High-P diet } \\
\hline Ca (mg/g dry weight) & $0.65 \pm 0.05$ & $13.07 \pm 2.31^{*}$ \\
$\mathrm{Mg}(\mathrm{mg} / \mathrm{g}$ dry weight $)$ & $0.97 \pm 0.04$ & $1.86 \pm 0.16^{*}$ \\
$\mathrm{P}(\mathrm{mg} / \mathrm{g}$ dry weight $)$ & $13.52 \pm 0.73$ & $20.54 \pm 1.37^{*}$
\end{tabular}

Values are means $\pm \mathrm{SD}, n=5$ per group.

*Significantly different from the control diet $(p<0.05)$.

\section{Results}

\section{Body weight}

Initial and final body weights are shown in Table 2 . There were no significant differences in initial and final body weights between the two groups.

\section{Kidney mineral concentration}

Kidney mineral concentration is shown in Table 3. Kidney $\mathrm{Ca}, \mathrm{Mg}$ and $\mathrm{P}$ concentrations were significantly increased in rats fed on the high-P diet compared with the rats fed on the control diet.
Table 4. Incidence of $\mathrm{Ca}$ deposits and osteopontin expression in rats fed on control and high-P diet.

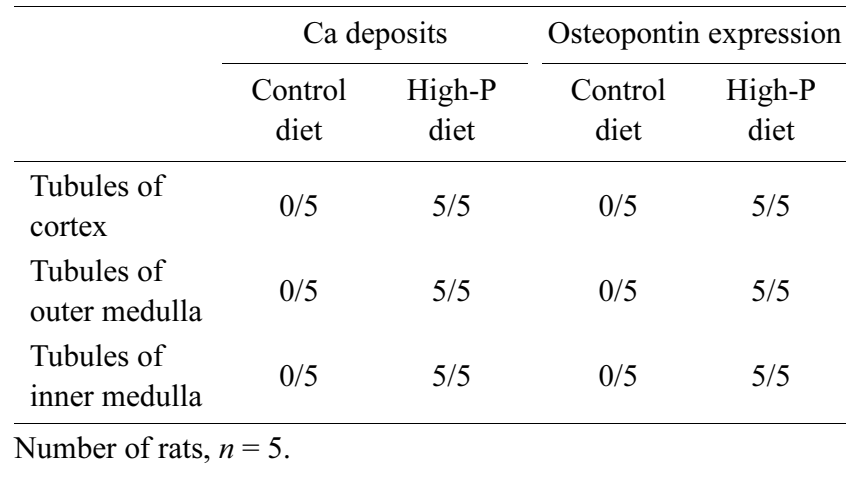

\section{Ca deposits and osteopontin expression in kidney}

The results of histological examination are shown in Table 4, Fig. 1 and Fig. 2. Ca deposits and osteopontin expression were observed in all rats fed on the high-P diet. $\mathrm{Ca}$ deposits were observed in the tubules of the cortex, outer medulla and inner medulla of rats fed on the high-P diet. Osteopontin expression was also seen in the tubules of the cortex, outer medulla and inner medulla of rats fed on the high-P diet. It is noteworthy that expression of osteopontin in rats fed on the high-P diet was observed in the almost same regions as the $\mathrm{Ca}$ deposits. In the rats fed on the control diet, no evidence of $\mathrm{Ca}$ deposits and osteopontin expression was observed in the tubules of the cortex, outer medulla or inner medulla.
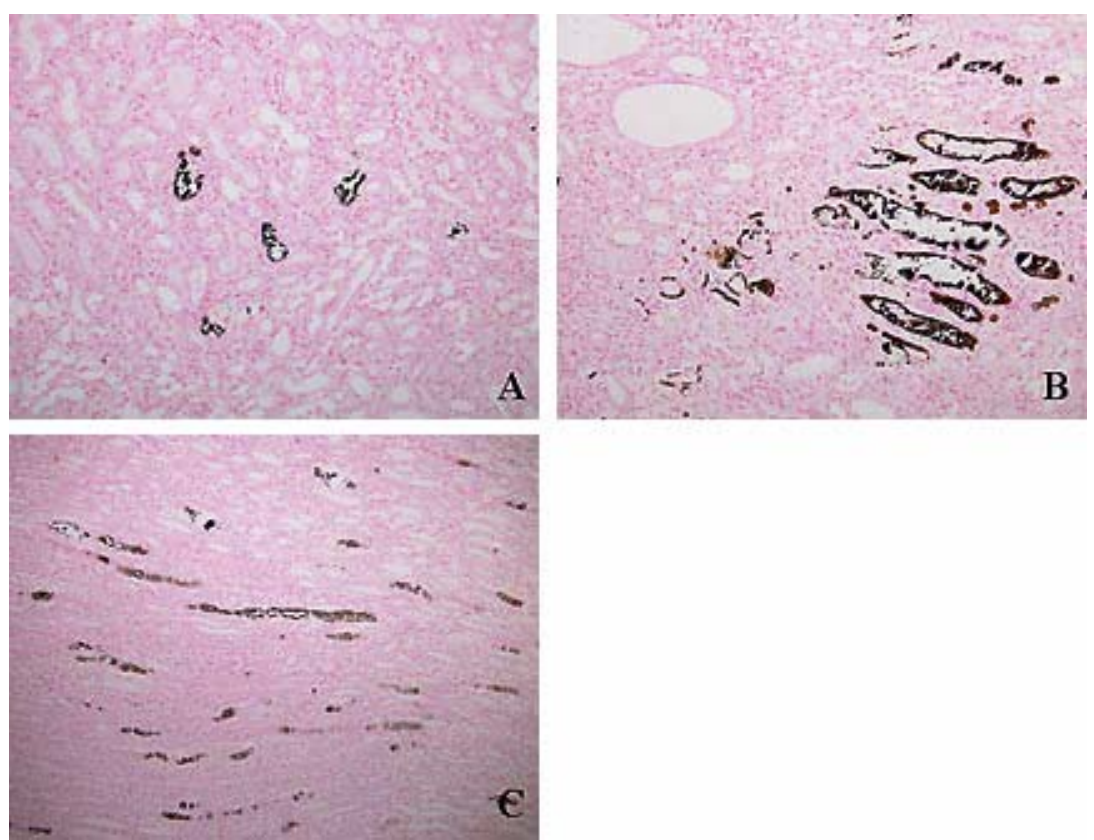

Fig. 1. Von Kossa staining for Ca deposits in kidney from high-P group showing Ca deposits in tubules of the cortex, outer medulla and inner medulla as black granules. A: Cortex region. B: Outer medulla region. C: Inner medulla region. Original magnification, $\times 200$. 


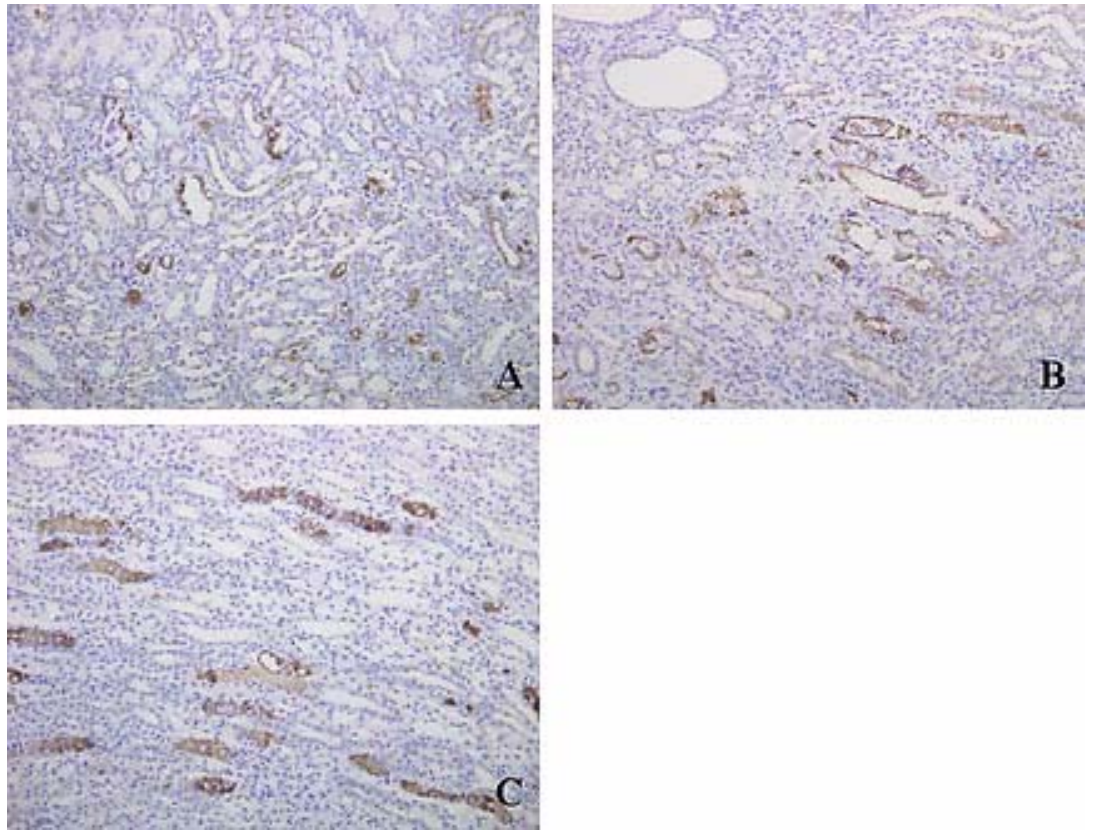

Fig. 2. Immunohistochemical staining for osteopontin in kidney from high-P group showing osteopontin expression in tubules of the cortex, outer medulla and inner medulla as brown granules. A: Cortex region. B: Outer medulla region. C: Inner medulla region. Original magnification, $\times 200$.

\section{Discussion}

This study showed that kidney $\mathrm{Ca}, \mathrm{Mg}$ and $\mathrm{P}$ concentrations were elevated in rats fed on the high-P diet, and that on histological examination (von Kossa staining), Ca deposits were observed in the tubules of the cortex, outer medulla and inner medulla in rats fed on the high-P diet. These findings are similar to the results of previous studies [1-5], and it was demonstrated that high-P diet induces nephrocalcinosis.

The mechanism responsible for onset of nephrocalcinosis, the understanding of which is crucial for prevention of the disorder, remains unclear. We hypothesized that during the formation of $\mathrm{Ca}$ deposits, matrix ingredients initially act as a nucleus, around which $\mathrm{Ca}$ deposits form. Matrix components play an important role in the formation of $\mathrm{Ca}$ deposits with high-P diet. On the other hand, the Ca deposits are mainly composed $\mathrm{Ca}$ and $\mathrm{P}$, as indicated by $\mathrm{X}$-ray microanalysis $[6,7]$, thus indicating that the Ca deposits in kidney are present as hydroxyapatite. Furthermore, we found that the high-P diet induces deposition of hydroxyapatite [7, $8]$. Based on these observations, we focused on osteopontin as matrix components in Ca deposits; osteopontin is able to bind to hydroxyapatite [9]. Therefore, immunohistochemical staining was used to observed the expression of osteopontin in the kidneys of rats fed on the high-P diet. Consequently, osteopontin was expressed in the tubules of the cortex, outer medulla and inner medulla in rats fed on the high-P diet. We also found that the locations of osteopontin expression and the $\mathrm{Ca}$ deposits overlapped. These observations suggest that osteopontin is present in the $\mathrm{Ca}$ deposits induced by high-P diet. Moreover, osteopontin in renal tubule is presumably involved in the formation of the $\mathrm{Ca}$ deposits induced by high-P diet. As described above, although osteopontin in rats fed on the high-P diet was localized in the tubules of the cortex, outer medulla and inner medulla, expression of this protein was not observed in the tubules of rats fed on the control diet. These results suggest that the high-P diet stimulate osteopontin expression in the renal tubules.

At present, the details of the relationship between osteopontin expression and the onset of nephrocalcinosis in rats fed on the high-P diet remain unclear. Therefore, further studies are needed to elucidate this point. However, we believe the results of this study provide valuable information into understanding the mechanisms responsible for onset of nephrocalcinosis.

In conclusion, this study investigated the effect of high-P diet on osteopontin expression in kidney. Ca deposits were observed in the tubules of the cortex, outer medulla and inner medulla of rats fed on the high-P diet. Osteopontin expression was confirmed in the tubules of the cortex, outer medulla and inner medulla of rats fed on the high-P diet. The locations of osteopontin expression and the Ca deposits in the kidney overlapped. The results of this study suggest that high-P diet induces osteopontin expression in the renal tubules. Moreover, osteopontin in the renal tubules presumably play a role as matrix components in the formation of $\mathrm{Ca}$ deposits with high-P diet administration. 


\section{References}

[1] Zalups, R.K., Haase, P., and Philbrick, D.J.: Phosphate and the development of nephrocalcinosis in rats fed diets containing alpha protein. Am. J. Pathol., 113, 95-106, 1983.

[2] Ritskes-Hoitinga, J., Lemmens, A.G., Danse, L.H.J.C., and Beynen, A.C.: Phosphorus-induced nephrocalcinosis and kidney function in female rats. J. Nutr., 119, 1423-1431, 1989.

[3] Matsuzaki, H., Masuyama, R., Uehara, M., Nakamura, K., and Suzuki, K.: Greater effect of dietary potassium tripolyphosphate than of potassium dihydrogenphosphate on the nephrocalcinosis and proximal tubular function in female rats from the intake of a high-phosphorus diet. Biosci. Biotechnol. Biochem., 65, 928-934, 2001.

[4] Cockell, K.A., L'Abbe, M.R., and Belonje, B.: The concentrations and ratio of dietary calcium and phosphorus influence development of nephrocalcinosis in female rats. J. Nutr., 132, 252-256, 2002.

[5] Matsuzaki, H., Masuyama, R., Uehara, M., Nakamura, K., and Suzuki, K.: Effects of simultaneous increases in dietary phosphorus and magnesium concentrations on nephrocalcinosis and kidney function in female rats. Magnes. Res., 17, 14-19, 2004.

[6] Stonard, M.D., Samuels, D.M., and Lock, E.A.: The pathogenesis and effect on renal function of nephrocalcinosis induced by different diets in female rats. Food Chem. Toxicol.,
22, 139-146, 1984.

[7] Liu, Q-L., Sato, S., Kishikawa, T., Matsuzaki, H., and Yamanaka, N.: Effectiveness of a traditional Chinese medicine, Wulingsan, in suppressing the development of nephrocalcinosis induced by a high phosphorus diet in young rats. Med. Electron Microsc., 34, 103-114, 2001.

[8] Matsuzaki, H., Uehara, M., Suzuki, K., Liu, Q-L., Sato, S., Kanke, Y., and Goto, S.: High phosphorus diet rapidly induces nephrocalcinosis and proximal tubular injury in rats. J. Nutr. Sci. Vitaminol., 43, 627-641, 1997.

[9] Oldberg, A., Franzen, A., and Heinegard, D.: Cloning and sequence analysis of rat bone sialoprotein (osteopontin) cDNA reveals an Arg-Gly-Asp cell-binding sequence. Proc. Natl. Acad. Sci. U.S.A., 83, 8819-8823, 1986.

[10] Reeves, P.G., Nielsen, F.H., and Fahey, G.C., Jr.: AIN-93 purified diets for laboratory rodents: Final report of the American Institute of Nutrition ad hoc writing committee on the reformulation of the AIN-76A rodent diet. J. Nutr., 123, 1939-1951, 1993.

[11] Gimblet, E.G., Marney, A.F., and Bonsnes, R.W.: Determination of calcium and magnesium in serum, urine, diet, and stool by atomic absorption spectrophotometry. Clin. Chem., 13, 204$214,1967$.

[12] Gomori, G.: A modification of the colorimetric phosphorus determination for use with the photoelectric colorimeter. $J$. Lab. Clin. Med., 27, 955-960, 1942. 